\title{
COMPUTING THE EQUATIONS OF A VARIETY
}

\author{
MICHELA BRUNDU AND MIKE STILLMAN
}

\begin{abstract}
Let $X \subset \mathbb{P}^{n}$ be a projective variety or subscheme, and let $\mathscr{F}$ be an invertible sheaf on $X$. A set of global sections of $\mathscr{F}$ determines a map from a Zariski open subset of $X$ to $\mathbb{P}^{r}$. The purpose of this paper is to find, given $X$ and $\mathscr{F}$, the homogeneous ideal defining the image in $\mathbb{P}^{r}$ of this rational map. We present algorithms to compute the ideal of the image. These algorithms can be implemented using only the computation of Gröbner bases and syzygies, and they have been implemented in our computer algebra system Macaulay. Our methods generalize to include the case when $X$ is an arbitrary projective scheme and $\mathscr{F}$ is generically invertible.
\end{abstract}

\section{INTRODUCTION}

Let $X \subset \mathbb{P}^{n}$ be a projective variety or subscheme, and let $\mathscr{F}$ be an invertible sheaf on $X$. A set of global sections of $\mathscr{F}$ determines a map from a Zariski open subset of $X$ to $\mathbb{P}^{r}$. The purpose of this paper is to find, given $X$ and $\mathscr{F}$, the homogeneous ideal defining the image in $\mathbb{P}^{r}$ of this rational map. Throughout this paper, we always deal with rational maps from $X$ to projective space.

The first question is how should one represent an invertible sheaf $\mathscr{F}$ on $X$ ? For general $X$, we can represent $\mathscr{F}$ by local data. In fact, choose an affine open cover $\left\{U_{i}\right\}$ of $X$. A sheaf of $\mathscr{O}_{X}$-modules $\mathscr{F}$ is determined by a module $\mathscr{F}\left(U_{i}\right)$ on $U_{i}$ and by patching maps on each $U_{i} \cap U_{j}$.

Since $X$ is projective, there is an alternate representation of a sheaf of $\mathscr{O}_{X^{-}}$ modules $\mathscr{F}$ as a graded $R$-module, where $R=R(X)$ is the homogeneous coordinate ring of $X$. This representation has a number of advantages. It requires less bookkeeping, contains more information, and algorithms using this representation are simpler. We use the module representation in this paper. In the first section, we recall the relationship between sheaves and graded modules. The remainder of the paper mostly uses the terminology of modules.

The image of the above rational map is easiest to compute if you know an open set over which $\mathscr{F}$ is free. In this case, sections correspond over this open

Received by the editors January 3, 1991.

1991 Mathematics Subject Classification. Primary 13F20; secondary 13-04, 14C20, 14 F05.

Key words and phrases. Line bundle, invertible sheaf, rational map, computing, Gröbner bases, symmetric algebra, local cohomology.

The second author was partially supported by the U.S. Army Research Office through the Mathematical Sciences Institute of Cornell University, and by NSF grants CCR-89-01061 and DMS-8802276. 
set to elements of $R(X)$, and the image ring $R(Y)$ is the subring of $R(X)$ generated by these elements.

More generally, suppose that $\mathscr{F}$ is an invertible sheaf on $X$, but that it is not feasible to compute an open set over which $\mathscr{F}$ is free. Recall that the projective bundle of $\mathscr{F}$ is

$$
\mathbb{P}(\mathscr{F}):=\operatorname{Proj} \mathscr{O}_{X} \oplus \mathscr{F} \oplus S^{2}(\mathscr{F}) \oplus \cdots,
$$

where $S^{b}(\mathscr{F})$ is the $b$ th symmetric power of $\mathscr{F}$ (see [Ha, Chapter II, §7] for definitions and details). If $\mathscr{F}$ is invertible, then the natural projection $\mathbb{P}(\mathscr{F}) \rightarrow X$ is an isomorphism. Moreover, global sections of $\mathscr{F}$ give rise to global sections of $\mathscr{O}_{\mathbf{P}(\mathscr{F})}(1)$. The image of $\mathbb{P}(\mathscr{F})$ under the map corresponding to these sections is the same as the image of $X$. These global sections are elements of the bigraded coordinate ring of $\mathbb{P}(\mathscr{F})$, and $R(Y)$ is the subring generated by these elements. In $\S 2$, we describe these techniques, and in $\S 3$, we present corresponding algorithms.

The methods we use generalize in several ways. First, although the most interesting case is when $R$ is an integral domain, the technique we use does not assume this. In fact, $R$ can be both nonreduced and reducible. To find the coordinate ring of the image $Y$ of the map above, it suffices to only consider what happens on a dense Zariski open set of $X$. In particular, the questions we ask above all make sense if $\mathscr{F}$ is only invertible on a dense open subset of $X$. This is the level of generaiity in which we prove our main results in $\S 2$. Finally, one can generalize to the case when $\mathscr{F}$ is either locally free of rank $r$, or generically locally free of rank $r$. In this case one obtains a rational map from $\mathbb{P}(\mathscr{F})$ to $\mathbb{P}^{r}$. The image can be found using the same methods.

In order to apply these results, one must be able to produce modules corresponding to invertible sheaves. There are two useful methods for finding invertible sheaves. An ideal $J \subset R$ which is locally generated by a nonzero divisor of $R$ defines an effective divisor $D=Z(J)$ on $X$, and a corresponding invertible sheaf $\mathscr{O}_{X}(D)$. Since the sheaf associated to $J$ is $\mathscr{O}_{X}(-D)$ (see the next section for the relationship between modules and sheaves), and $\mathscr{O}_{X}(D)=\operatorname{Hom}_{\mathscr{O}_{X}}\left(\mathscr{O}_{X}(-D), \mathscr{O}_{X}\right)$, it follows that the module $\operatorname{Hom}_{R}(J, R)$ represents $\mathscr{O}_{X}(D)$. In this case, it is possible to find the image of $X^{\cdot}$ in $\mathbb{P}^{r}$ with less computation. Perhaps the most important sheaf on any projective scheme $X$ is the canonical sheaf $K_{X}$. If $X$ is smooth (or, more generally, locally Gorenstein), then $K_{X}$ is invertible. A module corresponding to this sheaf can be found by computing Ext modules. We describe both of these methods in $\S 4$.

The algorithms presented here have been implemented in the computer algebra system Macaulay [BS], and will soon be distributed with that system.

We use the following notation. $X \subset \mathbb{P}^{n}$ is a projective variety or subscheme whose homogeneous coordinate ring $R(X)=R=S / I$, where $S=$ $k\left[x_{0}, \ldots, x_{n}\right]$, and $k$ is a field. $m=\left(x_{0}, \ldots, x_{n}\right) \subset R$ is the homogeneous maximal ideal of $R . M$ is always a finitely generated graded $R$-module, and $\widetilde{M}$ is its sheafification on $X$ or on $\mathbb{P}^{n}$. Throughout the paper, $\alpha_{0}, \ldots, \alpha_{r}$ are degree zero elements of $M$. As defined in $\S 2, \phi: X \rightarrow \mathbb{P}^{r}$ is the rational map which these elements determine, $Y \subset \mathbb{P}^{r}$ is the closure of the image of $\phi$, and $R(Y)$ is its homogeneous coordinate ring.

We would like to thank David Eisenbud for many helpful discussions. 


\section{Preliminaries}

Modules and their sheafification. We recall the relationship between graded modules and coherent sheaves on projective space. For more details, see [Ha] or [FAC]. In the latter, Serre describes (implicitly) the relationship between local cohomology and sheaf cohomology, which is the basis of most computations of sheaf cohomology. For more details about computing sheaf cohomology, see [ES].

Let $R=S / I$ be a graded ring, where $S=k\left[x_{0}, \ldots, x_{n}\right]$, and let $X \subset \mathbb{P}^{n}$ be the corresponding subscheme or subvariety. Given a graded $R$-module $M$, the sheafification $\widetilde{M}$ of $M$ is a sheaf on $X$ whose fiber over any homogeneous prime ideal $P \neq\left(x_{0}, \ldots, x_{n}\right)$ is the homogeneous localization $M_{(P)}$ consisting of all elements of degree zero in the localization $M_{P}$.

A sheaf $\mathscr{F}$ of $\mathscr{O}_{X}$-modules on $X$ is coherent if there is a finitely generated graded $R$-module $M$ whose sheafification is $\mathscr{F}$.

If $\mathscr{F}$ is a coherent sheaf on $X$, define

$$
\Gamma_{*}(\mathscr{F}):=\bigoplus_{d \in \mathbb{Z}} H^{0}(X, \mathscr{F}(d)),
$$

where $H^{0}(X, \mathscr{F}(d))$ is the vector space of global sections of the sheaf $\mathscr{F}(d)$. $\Gamma_{*}(\mathscr{F})$ is a graded $R$-module.

The operations of sheafification and taking global sections are inverses to each other in the following sense. There is a natural (graded) homomorphism

$$
\alpha: M \rightarrow \Gamma_{*}(\tilde{M}) .
$$

This map is an isomorphism in high degrees, i.e. $\alpha_{d}$ is an isomorphism for $d \gg$ 0 . Conversely, the natural map $\beta: \widetilde{\Gamma_{*}(\mathscr{F})} \rightarrow \mathscr{F}$ is an isomorphism. It follows that two different graded modules $M$ and $N$ have the same sheafification if and only if $M_{\geq d} \cong N_{\geq d}$ for some $d \gg 0$, where $M_{\geq d}:=M_{d} \oplus M_{d+1} \oplus \cdots$ is the truncation of $M$.

The module of global sections, $\Gamma_{*}(\mathscr{F})$, is not always finitely generated, since it can be nonzero in all negative degrees. The truncation $\Gamma_{*}(\mathscr{F})_{\geq d}$ is finitely generated for every $d$.

For many problems, it is necessary to choose a basis for the vector space of all sections of $\widetilde{M}$. The natural map $\alpha_{0}: M_{0} \rightarrow H^{0}(\widetilde{M})$ is often not surjective. In order to find a basis of $H^{0}(\widetilde{M})$, it suffices to compute $\Gamma_{*}(\widetilde{M})$. All of the ideas necessary to do this appear in Serre [FAC]. For complete algorithms, see [ES].

The coherent sheaf $\widetilde{M}$ is called locally free of rank $r$ if for every homogeneous prime ideal $P \subset R$, where $P \neq\left(x_{0}, \ldots, x_{n}\right)$, the homogeneous localization $M_{(P)}$ is isomorphic to $\left(R_{(P)}\right)^{r}$. If $\widetilde{M}$ is locally free of rank one, $\widetilde{M}$ is called an invertible sheaf, or equivalently, a line bundle. We also say that $M$ is locally free of rank one, or invertible. A graded $R$-module $M$ is invertible if and only if there exist non-zero-divisors $h_{1}, \ldots, h_{N} \in R$ such that $M_{h} \cong R_{h}$ and $X=\bigcup_{i=1}^{N} X-Z\left(h_{i}\right)$. If $\widetilde{M}$ is locally free and if every irreducible component of $X$ has dimension at least one, then the module $\Gamma_{*}(\widetilde{M})$ is finitely generated. 
Using Fitting ideals, it is relatively easy to determine if a module corresponds to a locally free sheaf. Let

$$
G \stackrel{A}{\rightarrow} F \rightarrow M \rightarrow 0
$$

be a presentation of $M$, where $F$ and $G$ are graded free $R$-modules, and $A$ is a $\operatorname{rank} F \times \operatorname{rank} G$ matrix with entries in $R$. The $j$ th Fitting ideal of $M$, denoted $I_{j}(M)$, is the ideal of $R$ generated by the $(\operatorname{rank} F-j) \times(\operatorname{rank} F-j)$ minors of $A$. It is easy to check that this definition is independent of the presentation of $M$. With these definitions, $\widetilde{M}$ is locally free of rank $r$ if and only if $\sqrt{I_{r}(M)}=(1)$ and $I_{r-1}(M)=0$ (see [Eis] for basic information about Fitting ideals).

Local cohomology. We use local cohomology to relate $M$ and $\Gamma_{*}(\widetilde{M})$. Recall that given a Noetherian graded ring $R$, a homogeneous ideal $m$, and a graded $R$-module $M$, the $i$ th local cohomology of $M$ with respect to $m$ is

$$
H_{m}^{i}(M):=\underline{\lim _{\longrightarrow}} \operatorname{Ext}_{R}^{i}\left(R / m^{d}, M\right),
$$

for any $i \geq 0$, where the limit is over all $d \geq 0$. Since

$$
\operatorname{Ext}_{R}^{0}\left(R / m^{d}, M\right)=\operatorname{Hom}_{R}\left(R / m^{d}, M\right)=\left\{x \in M \mid m^{d} x=0\right\},
$$

it follows that

$$
H_{m}^{0}(M)=\left\{x \in M \mid m^{d} x=0 \text { for some } d\right\} .
$$

We shall also use the following alternate notation. If $N \subset M$ is a submodule and $J \subset R$ is an ideal, define

$$
\left(N:_{M} J^{\infty}\right):=\left\{x \in M \mid J^{d} x \subset N \text { for some } d\right\} .
$$

Therefore, $H_{m}^{0}(M)=\left(0::_{M} m^{\infty}\right)$.

Local cohomology is related to sheaf cohomology by the following.

Proposition 1. Let $R=k\left[x_{0}, \ldots, x_{n}\right] / I$ be a graded ring, and let $X \subset \mathbb{P}^{n}$ be the corresponding subscheme. If $m=\left(x_{0}, \ldots, x_{n}\right)$ is the irrelevant maximal ideal and $M$ is a graded $R$-module, then

(1) there is an exact sequence

$$
0 \rightarrow H_{m}^{0}(M) \rightarrow M \stackrel{\alpha}{\rightarrow} \Gamma_{*}(\widetilde{M}) \rightarrow H_{m}^{1}(M) \rightarrow 0,
$$

(2) there are isomorphisms

$$
H_{m}^{i}(M) \cong \bigoplus_{\alpha \in \mathbb{Z}} H^{i-1}(X, \widetilde{M}(\alpha)) \quad \text { for all } i \geq 2 .
$$

This standard fact follows using local cohomology of sheaves. It also follows from Čech cohomology (again, this appears in Serre's [FAC] paper): For any $i \geq 0$,

$$
H^{i}(X, \widetilde{M}) \cong \underset{\lim }{\longrightarrow} \operatorname{Ext}_{S}^{i}\left(\left(x_{0}^{d}, \ldots, x_{n}^{d}\right), M\right)_{0},
$$

where the limit is over all $d \geq 0$, and the subscript zero refers to the degree zero part of this module.

For $i=0$, this formula gives a method of computing $\Gamma_{*}(\widetilde{M})$. 
Proposition 2. Let $R=S / I$ be a graded ring all of whose associated prime ideals have (Krull-)dimension $\geq 2$.

(1) For sufficiently large $k$,

$$
\Gamma_{*}(\widetilde{M}) \cong \operatorname{Hom}_{R}\left(\left(x_{0}^{k}, \ldots, x_{n}^{k}\right), M\right) .
$$

(2) If $\operatorname{depth}_{m} R \geq 2$, and $J$ is an ideal of $R$, then

$$
\Gamma_{*}(\widetilde{J})=\left(J:\left(x_{0}, \ldots, x_{n}\right)^{\infty}\right) .
$$

The $k$ in the first statement can be determined from a finite free resolution of $M$ as an $S$-module, and the second statement can be generalized. See [ES] for details.

Example 3. If $X \subset \mathbb{P}^{n}$ is a projective scheme and $f \in S$ is a nonzero divisor on $R$, then if $J=(f) \subset R$ and $L=\left(\operatorname{Hom}_{R}(J, R)\right)^{\sim}$, then $L=(R(\operatorname{deg} f))^{\sim}$. If $\operatorname{depth}(R) \geq 2$, then $\Gamma_{*}(L)=\operatorname{Hom}_{R}(J, R) \cong R(\operatorname{deg} f)$. If $\operatorname{depth}(R)=1$, this is not usually the case. On the other hand, since $M=R(\operatorname{deg} f)$, we know that

$$
\Gamma_{*}(L)=\operatorname{Hom}_{R}\left(\left(x_{0}^{d}, \ldots, x_{n}^{d}\right), R\right)(\operatorname{deg} f)
$$

for sufficiently large $d$.

The symmetric algebra. The symmetric algebra of a module plays a central role in our work. Let $M$ be a finitely generated graded $R$-module, where $R$ is a graded ring. For any positive integer $b$, we denote the $b$ th symmetric power of $M$ over $R$ by $S^{b} M$. The symmetric algebra of $M$ is $S^{*} M=\bigoplus_{b \geq 0} S^{b} M$. This is finitely generated as an $R$-algebra. Choose a set of homogeneous generators of $M$ having degrees $d_{1}, \ldots, d_{p}$ respectively. Let

$$
G \stackrel{A}{\rightarrow} \bigoplus_{i=1}^{p} R\left(-d_{i}\right) \rightarrow M \rightarrow 0
$$

be the corresponding (graded) presentation of $M$, where $G$ is a graded free $R$-module and $A$ is a matrix with entries in $R$. As usual, we denote by $R(-d)$ the graded $R$-module $R$ having its generator in degree $d$. This presentation of $M$ gives rise to the following well-known presentation of $S^{*} M$.

Proposition 4. With notation as above, $S^{*} M=R\left[y_{1}, \ldots, y_{p}\right] / L$, where $L$ is the ideal generated by the entries of the vector $\left(y_{1}, \ldots, y_{p}\right) A$.

For general information about symmetric algebras, see [HSV].

Notice that $S^{*} M$ is bigraded. The $(a, b)$ bigraded part of $S^{*} M$ is $\left(S^{*} M\right)_{(a, b)}=S^{b}(M)_{a}$, where the $a$-degree of $S^{b} M$ is induced from the grading of the $b$ th tensor product $M \otimes \cdots \otimes M$. In terms of the above presentation, if $R=k\left[x_{0}, \ldots, x_{n}\right] / I$, then $\operatorname{deg} x_{i}=(1,0)$ and $\operatorname{deg} y_{i}=\left(d_{i}, 1\right)$.

\section{The image of a RATIONAL MAP to PROJECTIVE SPACE}

Let $X \subset \mathbb{P}^{n}$ be a subscheme and let $R=k\left[x_{0}, \ldots, x_{n}\right] / I$ be its homogeneous coordinate ring. Let $M$ be a graded $R$-module. If $M$ is invertible, then a set of elements of $M_{0}$ defines a rational map to projective space. In this section, we present an algorithm for finding equations for the image of this map. 
The method we use also applies to modules which are generically invertible. A graded module $M$ is called generically invertible if there is a homogeneous nonzero-divisor $h \in R$ of positive degree such that $M_{h} \cong R_{h}$. This is equivalent to the geometric condition that $\widetilde{M}$ is invertible on the dense open subset $X-Z(h)$ of $X$. In the special case when $M=J$ is an ideal of $R$, then $J$ is generically invertible if and only if $J$ contains a non-zero-divisor of $R$. More generally, recall that if $R$ is not an integral domain, the torsion submodule $T_{R}(N)$ of an $R$-module $N$ is

$T_{R}(N):=\{m \in N \mid$ there is a non-zero-divisor $h$ of $R$ such that $h m=0\}$.

Lemma 1. Let $M$ be a graded $R$-module. $M$ is generically invertible if and only if $M / T_{R}(M)$ is isomorphic to $J(e)$ for an ideal $J \subset R$ containing a (homogeneous) non-zero-divisor of degree $e \geq 1$.

Furthermore, if $M$ is generically invertible, and $h \in R$ is a homogeneous non-zero-divisor of positive degree such that $M_{h} \cong R_{h}$, then there exists an ideal $J \subset R$, depending on $h$, such that $M / T_{R}(M) \cong J\left(\operatorname{deg} h^{N}\right)$, and $h^{N} \in J$, for some positive integer $N$.

Recall that $J(e)$ is the ideal $J$ with the new grading $J(e)_{d}=J_{e+d}$.

Proof. If $M / T_{R}(M) \cong J$ and $h \in J$ is a non-zero-divisor, then $M_{h} \cong J_{h}=R_{h}$, so $M$ is generically invertible.

Conversely, assume $M$ is generically invertible and $\psi_{h}: M_{h} \rightarrow R_{h}$ is an isomorphism. Choose a set of generators $m_{1}, \ldots, m_{p}$ for $M$, such that $\psi_{h}\left(m_{1} / h^{N}\right)=1$ and $\psi_{h}\left(m_{i}\right)=g_{i} / h^{N}$ for $g_{i} \in R$, where $N$ is an integer. Define $\psi: M \rightarrow R\left(\operatorname{deg} h^{N}\right)$ by $\psi\left(m_{i}\right)=g_{i}$ for each $i$. Let $J=\left(g_{1}, \ldots, g_{p}\right)$. Clearly $\psi$ maps onto $J$, and the kernel of $\psi$ is $T_{R}(M)=\left\{m \in M \mid h^{e} m=\right.$ 0 for some $e\}$.

We recall the definition of the rational map to projective space defined by a set of sections of a (generically) invertible sheaf. Suppose that the graded $R$-module $M$ is generically invertible. Let $\alpha_{0}, \ldots, \alpha_{r} \in M_{0}$. These elements define a morphism $\phi$ from an open subset $U$ of $X$ to $\mathbb{P}^{r}$ in the following way. Let $h \in R$ be any non-zero-divisor such that $M_{h} \cong R_{h}$. Let $\gamma: M \rightarrow J(e)$ be the corresponding surjection to an ideal $J$, where $e=\operatorname{deg} h^{N}$ for some positive integer $N$. Let $\gamma_{i}:=\gamma\left(\alpha_{i}\right) \in J$. The elements $\gamma_{0}, \ldots, \gamma_{r} \in J$ are homogeneous of degree $e$. Define a morphism $\phi_{h}: U_{h} \rightarrow \mathbb{P}^{r}$ by setting

$$
\phi_{h}(x)=\left(\gamma_{0}(x) / h^{N}(x), \ldots, \gamma_{r}(x) / h^{N}(x)\right),
$$

where $U_{h}:=X-\left(Z(h) \cup Z\left(\gamma_{0}, \ldots, \gamma_{r}\right)\right)$. It is easy to check that these can be glued together to form a well-defined map $\phi: U \rightarrow \mathbb{P}^{r}$. This map is well defined with no assumptions about whether $R$ is reduced or irreducible. The open set $U$ is not necessarily dense in $X$; the $\gamma_{i}$ may all vanish on a specific component of $X$. Let $Y \subset \mathbb{P}^{r}$ be the closure of the image of $\phi$.

We use the following simple lemma in the proof of the next theorem.

Lemma 2. If $M$ is an $R$-module and $h \in R$ is a non-zero-divisor of $R$ such that $M_{h} \cong R_{h}$, then

$$
T_{R}\left(S^{*}(M)\right)=\left(0: S^{*} M h^{\infty}\right):=\left\{f \in S^{*} M \mid h^{N} f=0 \text { for some } N \gg 0\right\} .
$$


Proof. By hypothesis, it follows that $S^{*} M_{h} \cong R_{h}[z]$. Let $T=T_{R}\left(S^{*} M\right)$. The localization $T_{h}=0$, since $T_{h}$ is exactly the $R_{h}$-torsion submodule of $S^{*} M_{h}$, which is torsion free. Therefore some power of $h$ annihilates each element of $T$.

The following theorem and the corresponding algorithms in $\S 3$ are the main observations of this paper.

Theorem 3. Let $M$ be a graded $R$-module which is generically invertible. Choose a non-zero-divisor $h \in R$ such that $M_{h} \cong R_{h}$ and let $M \rightarrow J(e)$ be the corresponding surjective graded map to an ideal $J$. Let $\alpha_{0}, \ldots, \alpha_{r}$ be elements of $M$ of degree zero, and for each $i$, let $\gamma_{i} \in J$ be the image of $\alpha_{i}$ under this map. If $Y$ is the image of the morphism $\phi$ defined above, then

(a) $R(Y)$ is isomorphic to the sub-k-algebra of $R$ generated by $\gamma_{0}, \ldots, \gamma_{r}$,

(b) $R(Y)$ is isomorphic to the sub-k-algebra of the Rees algebra $R[J t]$ generated by $\gamma_{0} t, \ldots, \gamma_{r} t$, and

(c) $R(Y)$ is isomorphic to the sub-k-algebra of $S^{*}(M) / T_{R}\left(S^{*}(M)\right)$ generated by the images of $\alpha_{0}, \ldots, \alpha_{r}$.

Proof. First note that $\overline{\phi_{h}\left(U_{h}\right)}=Y$, since $U_{h}$ is dense in $U$. On the open set $U_{h}, \phi$ is represented by the polynomial map $\phi_{h}(x)=\left(\gamma_{0}(x), \ldots, \gamma_{r}(x)\right)$. Therefore, if $X^{\prime}$ is the closure of $U$ in $\mathbb{P}^{n}$, and $R^{\prime}=R\left(X^{\prime}\right)$ is its homogeneous coordinate ring, then $R(Y)$ is the subring of $R^{\prime}$ generated by $\gamma_{0}, \ldots, \gamma_{r}$.

Notice that $X^{\prime}$ is the union of the components of $X$ on which at least one $\gamma_{i}$ does not vanish identically. If $(0)=Q_{1} \cap \cdots \cap Q_{t}$ is the primary decomposition of (0) in $R$, and $R^{\prime}=R / Q_{1} \cap \cdots \cap Q_{s}$ for some $s \leq t$, then each $\gamma_{i} \in Q_{s+1} \cap \cdots \cap Q_{t}$. If $F\left(y_{0}, \ldots, y_{r}\right) \in k\left[y_{0}, \ldots, y_{r}\right]$ is homogeneous, and $F\left(\gamma_{0}, \ldots, \gamma_{r}\right) \in Q_{1} \cap \cdots \cap Q_{s}$, then since each $\gamma_{i} \in Q_{s+1} \cap \cdots \cap Q_{t}$, it follows that $F\left(\gamma_{0}, \ldots, \gamma_{r}\right)=0$. Therefore the subrings of $R$ and $R^{\prime}$ generated by $\gamma_{0}, \ldots, \gamma_{r}$ are isomorphic, proving (a).

Statement (b) follows since $\gamma_{0}, \ldots, \gamma_{r}$ are all homogeneous of the same degree. In this case, the subrings $k\left[\gamma_{0}, \ldots, \gamma_{r}\right] \subset R$ and $k\left[\gamma_{0} t, \ldots, \gamma_{r} t\right] \subset R[t]$ are isomorphic. Therefore $k\left[\gamma_{0} t, \ldots, \gamma_{r} t\right] \subset R[J t] \subset R[t]$ is isomorphic to $R(Y)$.

For (c), first notice that if $A$ is an arbitrary $k$-algebra, $x_{1}, \ldots, x_{m} \in A$, and $h \in A$ is a non-zero-divisor, then the subalgebras of $A$ and $A_{h}$ generated by $x_{1}, \ldots, x_{m} \in A$ are both isomorphic.

Since $h$ is a non-zero-divisor of $R[t], R(Y)$ is isomorphic to the sub-kalgebra of $R_{h}[t]$ generated by $\gamma_{0} t, \ldots, \gamma_{r} t$. There is an isomorphism $S^{*}(M)_{h}$ $\rightarrow S^{*}\left(R_{h}\right)=R_{h}[t]$. Under this isomorphism, $\alpha_{i} \mapsto \gamma_{i} t$ for each $i$. Therefore $R(Y)$ is isomorphic to the sub- $k$-algebra of $S^{*}(M)_{h}$ generated by $\alpha_{0}, \ldots, \alpha_{r}$.

By Lemma 2,

$$
T_{R}\left(S^{*}(M)\right)=\left(0: S^{*} M h^{\infty}\right) .
$$

Therefore, $h$ is a non-zero-divisor of the ring $S^{*} M / T\left(S^{*} M\right)$, and so the subalgebras of the two rings $S^{*} M / T\left(S^{*} M\right)$ and $S^{*} M / T\left(S^{*} M\right)_{h} \cong S^{*} M_{h}$ generated by the images of $\alpha_{0}, \ldots, \alpha_{r}$ are isomorphic, as desired.

If the ideal $J$ can be easily determined, then computing $R(Y)$ via Theorem $3(\mathrm{a})$ is the method of choice. If $J$ is computationally difficult to find, but the 
torsion of $S^{*} M$ can be identified (as in the line bundle case below), then part (c) of the theorem should be used.

If $M=J$ is an ideal containing a non-zero-divisor (i.e., $J$ is generically invertible), then we have the following diagram:

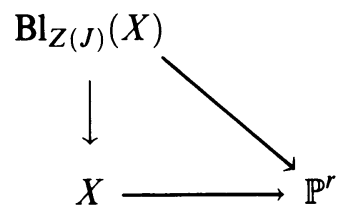

In this diagram $\mathrm{Bl}_{Z(J)}(X)$ is the blowup of $X$ along the zero scheme of $J$. Its bihomogeneous coordinate ring is $R[\mathrm{Jt}]$. The second part of the theorem states that these two maps to $\mathbb{P}^{r}$ have the same image (up to closure).

If $M$ is invertible, the torsion submodule of the symmetric module is easy to identify. In this case, the method of Theorem 3(c) works well in practice.

Corollary 4. Let $M$ be an invertible graded $R$-module, and let $R(Y)$ denote the homogeneous coordinate ring of the image $Y \subset \mathbb{P}^{r}$ of the rational map corresponding to the elements $\alpha_{0}, \ldots, \alpha_{r} \in M_{0} . R(Y)$ is isomorphic to the sub-k-algebra of $S^{*}(M) / H_{m}^{0}\left(S^{*} M\right)$ generated by the images of $\alpha_{0}, \ldots, \alpha_{r}$.

Proof. By the theorem, it suffices to show that

$$
T_{R}\left(S^{*} M\right)=\left(0: S^{*} M\left(x_{0}, \ldots, x_{n}\right)^{\infty}\right) \text {. }
$$

By hypothesis, there is an open cover $\left\{U_{i}\right\}_{\{i=1, \ldots, q\}}$ of $X, U_{i}=X-Z\left(h_{i}\right)$ for some non-zero-divisors $h_{i} \in R$, which trivializes $M$. In other words, $M_{h_{i}} \cong R_{h_{i}}$ for each $i$. By the above lemma, $T_{R}\left(S^{*} M\right)=\left(0:\left(h_{1}, \ldots, h_{q}\right)^{\infty}\right)$. Since the $U_{i}$ cover $X$, the ideal $\left(h_{1}, \ldots, h_{q}\right)$ is $m$-primary, where $m=$ $\left(x_{0}, \ldots, x_{n}\right) \subset R$. Therefore $T_{R}\left(S^{*} M\right)=\left(0: S^{*} M\left(x_{0}, \ldots, x_{n}\right)^{\infty}\right)$.

Corollary 4 can also be shown in the following way. The ring $R(Y)$ is the subring of $\bigoplus_{a, b} H^{0}\left(S^{b}(\widetilde{M})(a)\right)$ generated by the images of $\alpha_{0}, \ldots, \alpha_{r}$ under the image of the natural map $\alpha: M_{0} \rightarrow H^{0}(\widetilde{M})$. Since direct sums commute with local cohomology, there is an exact sequence

$$
0 \rightarrow H_{m}^{0}\left(S^{*} M\right) \rightarrow S^{*} M \rightarrow \bigoplus_{a, b} H^{0}\left(S^{b}(\widetilde{M})(a)\right)
$$

and therefore $R(Y)$ is isomorphic to the sub- $k$-algebra of $S^{*} M / H_{m}^{0}\left(S^{*} M\right)$ generated by $\alpha_{0}, \ldots, \alpha_{r}$.

Example 5. Let $S=k[a, b, c, d, e, f]$, and let $I$ be the ideal generated by the maximal minors of the matrix

$$
\left(\begin{array}{lll}
a & b & c \\
d & e & f
\end{array}\right)
$$

Let $R=S / I$, let $M=R(-1)^{2} /\left(\begin{array}{lll}a & b & c \\ d & e & f\end{array}\right)$, and let $E_{1}$ and $E_{2}$ be the two given generators of $M . R$ is the homogeneous coordinate ring of $\mathbb{P}^{1} \times \mathbb{P}^{2} \subset \mathbb{P}^{5}$, and up to a degree shift, $M$ is the canonical module of $R$.

The element $a \in R$ is a non-zero-divisor such that $M_{a} \cong R_{a}$, where the isomorphism is given by the map $E_{1} \mapsto-d / a, E_{2} \mapsto 1$. Since such an isomorphism exists for $h=a, b, c, d, e$, and $f$, the module $M$ is invertible. $M$ is 
isomorphic to a shift of the ideal $J=(a, d)$. Let

$$
\left\{a E_{1}, b E_{1}, c E_{1}, d E_{1}, e E_{1}, f E_{1}, a E_{2}, b E_{2}, c E_{2}\right\}
$$

be a basis for the degree zero part of $M$. The image of this basis in $J$ is

$$
\left\{a d, b d, c d, d^{2}, d e, d f, a^{2}, a b, a c\right\} \text {. }
$$

The sub- $k$-algebra of $R$ generated by these elements is easily computed using Gröbner bases. If $R(Y)$ denotes the resulting algebra, then

$$
R(Y) \cong k\left[X_{1}, \ldots, X_{9}\right] / L,
$$

where $L$ is the ideal generated by the maximal minors of the matrix

$$
\left(\begin{array}{llllll}
X_{1} & X_{2} & X_{3} & X_{4} & X_{5} & X_{6} \\
X_{7} & X_{8} & X_{9} & X_{1} & X_{2} & X_{3}
\end{array}\right)
$$

The symmetric algebra of $M$ is

$$
S^{*} M=R[x, y] /(a x+d y, b x+e y, c x+f y) .
$$

By computing the ideal quotient

$$
((a x+d y, b x+e y, c x+f y):(a, b, c, d, e, f)),
$$

or by inspection, one sees that $S^{*} M$ has no torsion. Using Theorem $3(\mathrm{c}), R(Y)$ is isomorphic to the sub- $k$-algebra of $S^{*} M$ generated by the nine elements $\{a x, b x, c x, d x, e x, f x, a y, b y, c y\}$. Using Gröbner bases, one calculates exactly the same presentation of $R(Y)$ as above.

The rational map $\phi: X \rightarrow \mathbb{P}^{8}$ corresponding to the nine elements of $J$ given above is defined everywhere except on $Z(J)$. The closure of the image of $\phi$ is the same as the image of the morphism from the blowup of $Z(J)$ to $\mathbb{P}^{8}$, by Theorem 3(b).

\section{AlgorithmS FOR FINDING THE IMAGE $R(Y)$}

We now describe two algorithms for finding the image ring $R(Y)$ corresponding to elements $\alpha_{0}, \ldots, \alpha_{r}$ of degree zero of the graded $R$-module $M . M$ is assumed to be generically invertible. The first method is based on Theorem 2.3(c), and the second method is based on Theorem 2.3(a).

These algorithms can be implemented by using Gröbner bases and syzygies (see [Buch 65, Buch 85, Ba], or [ES] for information about Gröbner bases).

More specifically, we assume there are subroutines for the following computations.

(1) Given an affine ring $R=k\left[x_{0}, \ldots, x_{n}\right] / I$, where $k$ is a field, and a set of elements $\alpha_{0}, \ldots, \alpha_{r}$, find a presentation

$$
k\left[\alpha_{0}, \ldots, \alpha_{r}\right] \cong k\left[y_{0}, \ldots, y_{r}\right] / J
$$

for the sub- $k$-algebra of $R$ which they generate.

(2) Given ideals $J, L \subset R$, find generators for the ideal $(J: L)=\{f \in R \mid$ $f L \subset J\}$ and the ideal $\left(J: L^{\infty}\right)=\left\{f \in R \mid f L^{N} \subset J\right.$, for some $N \gg$ $0\}$. 
The following notation applies to Algorithms 1 and 3 below. Let

$$
G \stackrel{A}{\rightarrow} F \rightarrow M \rightarrow 0
$$

be a (graded) presentation of $M$, where $F=\bigoplus_{i=1}^{p} R\left(-d_{i}\right)$. Let $\alpha_{0}, \ldots, \alpha_{r} \in$ $M_{0}$ be the images of $\left(v_{0}, \ldots, v_{r}\right)$, where each $v_{i} \in F$ is a column vector. Let $\vec{y}=\left(y_{1}, \ldots, y_{p}\right)$.

Algorithm 1. (Image of a map via symmetric algebras) input

a (graded) matrix $A$ over $R$ which presents the graded $R$-module $M$.

elements $v_{0}, \ldots, v_{r}$ of the row space $F$ of $A$, whose images in $M$ have degree zero. assumption $M$ is invertible.

output

the homogeneous coordinate ring $R(Y)$ of the image of the rational map corresponding to $v_{0}, \ldots, v_{r}$.

begin

set $J \subset R\left[y_{1}, \ldots, y_{p}\right]$ to be the ideal generated by the entries of the vector $\vec{y} A$.

(*) compute $T=S^{*} M / T_{R}\left(S^{*} M\right):=R\left[y_{1}, \ldots, y_{p}\right] /\left(J: m^{\infty}\right)$.

compute $R(Y):=$ the sub- $k$-algebra of $T$ generated by the elements $\vec{y} v_{0}, \ldots, \vec{y} v_{r}$. end.

If $M$ is generically invertible but not invertible, then the above algorithm needs to be modified to find the torsion in $S^{*} M$. Given a non-zero-divisor $h \in R$ such that $M_{h} \cong R_{h}$, we replace $\left(J: m^{\infty}\right)$ in the starred line with $\left(J: h^{\infty}\right)$.

In terms of Fitting ideals, $M$ is generically invertible if and only if $I_{0}(M)=$ (0) and $I_{1}(M)$ contains a non-zero-divisor of $R$. In this case, any non-zerodivisor $h \in I_{1}(M)$ satisfies $M_{h} \cong R_{h}$. The following proposition gives an algorithm for finding such an $h$ and an ideal $J \subset R$ such that $M / T_{R}(M) \cong J$.

Proposition 2. Let $M$ be a generically invertible graded $R$-module. Choose a (graded) presentation matrix $A: R^{b} \rightarrow R^{a} \rightarrow M \rightarrow 0$ such that the $(a-1) \times$ $(a-1)$ principal minor $\Delta$ of $A$ is a non-zero-divisor in $R$. If $J \subset R$ is the ideal generated by the $(a-1) \times(a-1)$ minors of $A$ involving the first $a-1$ columns of $A$, then there exists an isomorphism $M / T_{R}(M) \rightarrow J(\operatorname{deg} \Delta)$.

Proof. Let $\Delta_{i} \in J$ be the minor involving the first $a-1$ columns of $A$, but omitting the $i$ th row for $i=1, \ldots, a$. Therefore $J=\left(\Delta_{1}, \ldots, \Delta_{a-1}, \Delta\right)$ and $\Delta=\Delta_{a}$. Let $e_{1}, \ldots, e_{a} \in M$ be the images of the unit vectors of $R^{a}$ in $M$. By column operations on $A$ (i.e., Cramer's rule), there are equalities

$$
\Delta e_{i}=\Delta_{i} e_{a}
$$

for each $i=1, \ldots, a-1$. Furthermore, since every $a \times a$ minor of $A$ is zero and $\Delta$ is a non-zero-divisor, no nonzero element of $R$ annihilates $e_{a}$.

Localization by $\Delta$ determines an isomorphism $\psi_{\Delta}: M_{\Delta} \rightarrow R_{\Delta}$ defined by $e_{i} \mapsto \Delta_{i} / \Delta$ for $i=1, \ldots, a-1$, and $e_{a} \mapsto 1$. This map is well defined, since if $\sum_{i=1}^{a} \beta_{i} e_{i}=0$ in $M$ for $\beta_{i} \in R$, then $\sum_{i=1}^{a} \beta_{i} \Delta e_{i}=0$, and so $\left(\sum \beta_{i} \Delta_{i}\right) e_{a}=0$. Therefore $\sum \beta_{i} \Delta_{i}=0$, i.e., the image under $\psi_{\Delta}$ of $\sum_{i=1}^{a} \beta_{i} e_{i}$ is zero. Once the map is defined, it is easily seen to be an isomorphism.

Given this isomorphism, the proof of Lemma 2.1 produces the desired isomorphism $M / T_{R}(M) \rightarrow J(\operatorname{deg} \Delta)$. 
This proposition, and Theorem $2.3(\mathrm{a})$ immediately give the following algorithm.

Algorithm 3. (Image of a map via ideals)

input

a (graded) matrix $A$ over $R$ which presents the graded $R$-module $M$.

elements $v_{0}, \ldots, v_{r}$ of the row space $F$ of $A$, whose images in $M$ have degree zero.

assumption

$M$ is generically invertible.

output

the homogeneous coordinate ring $R(Y)$ of the image of the rational map corresponding to $v_{0}, \ldots, v_{r}$.

begin

Choose a new presentation matrix $B$ for $A$ by using row and column operations, so that the principal $(a-1) \times(a-1)$ minor $\Delta$ of $B$ is a non-zero-divisor of $R$. compute $\Delta_{i} \in R$, for $i=1, \ldots, a$, the $(a-1) \times(a-1)$ minor of the submatrix of $B$ consisting of the first $a-1$ columns of $B$, and omitting the $i$ th row of $B$. let $\vec{\Delta}:=\left(\Delta_{1}, \ldots, \Delta_{a}\right)$.

compute $R(Y):=$ the sub- $k$-algebra of $R$ generated by the elements $\vec{\Delta} v_{0}, \ldots, \vec{\Delta} v_{r}$. end.

To determine whether $\Delta$ is a non-zero-divisor of $R$, it suffices to compute $(0: \Delta)$; this ideal quotient is zero if and only if $\Delta$ is a non-zero-divisor.

\section{Constructing line BUNDles on A SCHEME}

Let $X \subset \mathbb{P}^{n}$ be the projective scheme corresponding to the graded ring $R=$ $S / I$, where $I \subset S=k\left[x_{0}, \ldots, x_{n}\right]$ is a homogeneous ideal.

One must be able to find line bundles on $X \subset \mathbb{P}^{n}$ in order to use the algorithms in the previous section. In this section we discuss two useful methods: effective divisors and canonical modules.

Effective divisors. By definition, an effective divisor on $X$ is a subscheme, $Z(J)$, of $X$ which is locally generated by a non-zero-divisor in $R$, i.e., the homogeneous ideal $J$ is invertible. In this case $M=\operatorname{Hom}_{R}(J, R)$ is also invertible. The sheafification of $M$ is the line bundle $\mathscr{O}_{X}(D)$ on $X$, where $D$ is the effective divisor $Z(J)$.

More generally, if $J \subset R$ is generically invertible and $M=\operatorname{Hom}(J, R)$, then $M$ is also generically invertible. In this case, $M$ is isomorphic to the ideal of the residual intersection of $Z(J)$ in $Z(f)$, where $f \in J$ is any nonzero-divisor.

Proposition 1. If $R$ is any ring (commutative with identity), and $f \in J$ is a non-zero-divisor of $R$, then

$$
\operatorname{Hom}_{R}(J, R) \cong(f): J .
$$

Proof. There is a natural map $\theta: \operatorname{Hom}_{R}(J, R) \rightarrow(f): J$, defined by $\theta(\alpha)=$ $\alpha(f)$. This is well defined since $\alpha(f) g=f \alpha(g) \in(f)$. Injectivity follows easily since $f$ is a non-zero-divisor. For surjectivity, let $\beta \in(f): J$. For each $g \in J$, there exists a unique $\gamma \in R$ such that $\beta g=f \gamma$. Uniqueness follows since $f$ is a non-zero-divisor. Define $\alpha(g):=\gamma$. Clearly $\alpha$ is a homomorphism, and $\theta(\alpha)=\alpha(f)=\beta$, as desired. 
The canonical bundle. If $X \subset \mathbb{P}^{n}$ is a smooth projective variety or more generally locally Gorenstein, and $K_{X}$ is the canonical line bundle on $X$, then we are able to find the module $K_{R}$ associated to $K_{X}$, by using the following well-known consequence of Serre duality.

Proposition 2. If $I \subset S=k\left[x_{0}, \ldots, x_{n}\right]$ is the homogeneous ideal of a smooth projective variety $X$ of codimension $c$, and $K_{X}$ is the canonical line bundle of $X$, then

$$
\bigoplus_{d \in \mathbb{Z}} H^{0}(X, K(d)) \cong \operatorname{Ext}^{c}(S / I, S(-n-1))
$$

is an isomorphism of graded $S$-modules.

This follows more generally whenever $X$ is locally Cohen-Macaulay, equidimensional of codimension $c$ : in this case, if $K_{X}$ is the dualizing sheaf of $X$, then Serre duality gives

$$
\left(\bigoplus_{d \in \mathbb{Z}} H^{n-c}\left(X, \mathscr{O}_{X}(d)\right)\right)^{\prime} \cong \bigoplus_{d \in \mathbb{Z}} H^{0}\left(X, K_{X}(d)\right),
$$

where the dual on the left is the $k$-dual of the graded module (see, for example, [Ha]). But (see [FAC])

$$
\left(\bigoplus_{d \in \mathbb{Z}} H^{n-c}\left(X, \mathscr{O}_{X}(d)\right)\right)^{\prime} \cong \operatorname{Ext}^{c}(S / I, S(-n-1)),
$$

giving the desired statement.

The module $\operatorname{Ext}^{c}(S / I, S)$ can be computed using Gröbner bases and syzygies.

\section{SOME EXAMPLES}

Let $C \subset \mathbb{P}^{3}$ be a smooth curve of genus $g$ and degree $d$. In this case, the canonical bundle is the sheafification of $\operatorname{Ext}^{2}(S / I, S(-4))$, where $S=$ $k[w, x, y, z]$, and $I \subset S$ is the homogeneous ideal of $C$. Set $R=S / I$ to be the homogeneous coordinate ring of $C$.

If $C$ is embedded as a projectively normal curve (i.e., $S / I$ is a normal domain), then the theorem of Hilbert and Burch implies that there is an $(n+1) \times n$ matrix $A$ with homogeneous polynomial entries such that the ideal $I$ is defined by the maximal minors of this matrix, and the finite free resolution of $S / I$ is

$$
0 \rightarrow \bigoplus_{i=1}^{n} S\left(-d_{i}\right) \stackrel{A}{\rightarrow} \bigoplus_{j=1}^{n+1} S\left(-e_{j}\right) \rightarrow S \rightarrow S / I \rightarrow 0 .
$$

Consequently, the canonical sheaf is the sheafification of the $R$-module

$$
\operatorname{Ext}^{2}(S / I, S(-4))=\bigoplus_{i=1}^{n} R\left(d_{i}-4\right) / \text { image } A^{t r} \text {. }
$$

Example 1. Let $C$ be a curve of genus 5 which is not trigonal, i.e., does not admit a 3-to-1 finite map to $\mathbb{P}^{1}$ (for definitions, see Hartshorne [Ha] or Fulton [Fu]). Using basic properties of curves, it is easy to see that $C$ can be embedded 
as a projectively normal curve $C$ of degree 7 in $\mathbb{P}^{3}$. The ideal $I$ of $C$ is generated by the $2 \times 2$ minors of a matrix

$$
A^{t r}=\left(\begin{array}{lll}
L_{1} & L_{2} & L_{3} \\
Q_{1} & Q_{2} & Q_{3}
\end{array}\right)
$$

where each $L_{i} \in S$ is a linear form and $Q_{i} \in S$ is a quadratic form. The canonical module is

$$
M=R \oplus R(1) / \text { image } A^{\text {tr }},
$$

where $R=S / I$. If the two unit vectors generating $M$ are denoted by $e_{1}$ and $e_{2}$, where $\operatorname{deg} e_{1}=0$ and $\operatorname{deg} e_{2}=-1$, then

$$
e_{1}, w e_{2}, x e_{2}, y e_{2}, z e_{2}
$$

form a basis for the global sections of the canonical bundle.

The symmetric module $S^{*} M$ of $M$ is

$$
S^{*} M=k[w, x, y, z]\left[e_{1}, e_{2}\right] / J,
$$

where

$$
J=I+\left(L_{1} e_{1}+Q_{1} e_{2}, L_{2} e_{1}+Q_{2} e_{2}, L_{3} e_{1}+Q_{3} e_{2}\right) .
$$

In this case, it is easy to see that $H_{m}^{0}\left(S^{*} M\right)=0$.

Therefore, the canonical curve, $C_{K}$, which is the image of the map $C \rightarrow \mathbb{P}^{4}$, defined by

$$
p \mapsto(v, w, x, y, z)=\left(e_{1}, w e_{2}, x e_{2}, y e_{2}, z e_{2}\right)(p),
$$

is defined by the ideal

$$
L=\left(L_{1} v+Q_{1}, L_{2} v+Q_{2}, L_{3} v+Q_{3}\right) .
$$

For example, one checks that, in $S^{*} M$,

$$
L_{1}\left(w e_{2}, x e_{2}, y e_{2}, z e_{2}\right) e_{1}+Q_{1}\left(w e_{2}, x e_{2}, y e_{2}, z e_{2}\right)=L_{1} e_{1} e_{2}+Q_{1} e_{2}^{2}=0 \text {. }
$$

Notice also that for each $i=1,2,3$, the canonical module $M$ is isomorphic to the ideal $J=\left(L_{i}, Q_{i}\right) \subset R$.

Example 2. Let $C \subset \mathbb{P}^{3}$ be a general curve of degree $d=10$ and genus $g=11$. It turns out that such a curve must be embedded as a projectively normal curve, whose ideal $I$ is defined by the $4 \times 4$ minors of a $5 \times 4$ matrix $A=\left(L_{i j}\right)$ of linear forms in $S=k\left[x_{0}, x_{1}, x_{2}, x_{3}\right]$. The canonical module of $C$ is

$$
R(1)^{4} / \text { image } A^{t r} \text {, }
$$

where $R=S / I$. Denote the corresponding generators of this module by $e_{0}, \ldots, e_{3}$. The sections of the canonical bundle are generated by the $16 \mathrm{sec}-$ tions $z_{i j}:=x_{i} e_{j}$ for $0 \leq i, j \leq 3$. These are not linearly independent; they have five relations:

$$
\sum_{j=0}^{3} L_{i j} e_{j}=0
$$

for each $i=1, \ldots, 5$. In any case, the ideal of the canonical embedding in $\mathbb{P}^{10}$ contains the 2-by-2 minors of the 4-by-4 matrix of linear forms $\left(z_{i j}\right)$ on $\mathbb{P}^{10}$. In fact, if $C$ is not embedded by the half-canonical series, then these 36 
minors generate the entire ideal. Therefore such canonical curves are generic sections of the determinantal variety of all 4-by-4 matrices of rank one.

Example 3. The theory of this paper also works if $M$ is locally free of rank $p>1$. For example, if $R=k[s, t]$ and $M=R\left(a_{1}\right) \oplus \cdots \oplus R\left(a_{p}\right)$, then the symmetric algebra is

$$
S^{*} M=k[s, t]\left[y_{1}, \ldots, y_{p}\right],
$$

where $\operatorname{deg} y_{i}=\left(-a_{i}, 1\right)$ for each $i$. The sections of $M_{0}$ are

$$
s^{a_{1}} y_{1}, s^{a_{1}-1} t y_{1}, \ldots, t^{a_{1}} y_{1}, \ldots, s^{a_{p}} y_{p}, s^{a_{p}-1} t y_{p}, \ldots, t^{a_{p}} y_{p} .
$$

The image of $\mathbb{P}\left(\mathscr{O}\left(a_{1}\right) \oplus \cdots \oplus \mathscr{O}\left(a_{p}\right)\right)$ under this polynomial map is exactly the rational normal scroll $S\left(a_{1}, \ldots, a_{p}\right)$.

More generally, if $M$ is locally free of rank $p$ on a scheme $X, \alpha_{0}, \ldots$, $\alpha_{r}$ are elements of $M_{0}$, and $Y$ is the image of $\mathbb{P}(\widetilde{M})$ under the corresponding sections of $\mathscr{O}_{\mathbf{P}(\widetilde{M})}$, then $R(Y)$ is isomorphic to the sub-k-algebra generated by the images of $\alpha_{0}, \ldots, \alpha_{r}$ in $S^{*}(M) / H_{m}^{0}\left(S^{*} M\right)$.

\section{REFERENCES}

[Ba] D. Bayer, The division algorithm and the Hilbert scheme, Ph.D. thesis, Harvard University, 1982.

[BS] D. Bayer and M. Stillman, Macaulay: A system for computation in algebraic geometry and commutative algebra, Source and object code available for Unix and Macintosh computers. Contact the authors, or ftp zariski.harvard.edu, Name: ftp, Password: any, cd Macaulay, binary, get M3.tar, quit, tar xf M3.tar.

[Buch 65] B. Buchberger, Ein Algorithmus zum Auffinden der Basiselemente des Restklassenringes nach einem nulldimensionalen Polynomideal, Ph.D. thesis, Universität Innsbruck, 1965.

[Buch 85] Gröbner bases: An algorithmic method in polynomial ideal theory, Multidimensional Systems Theory (N. K. Bose, ed.), Reidel, 1985, pp. 184-232.

[Eis] D. Eisenbud, Commutative algebra with a view toward algebraic geometry, Brandeis Lecture Notes, Brandeis Univ., Waltham, Mass., 1989.

[ES] D. Eisenbud and M. Stillman, Methods for computing in algebraic geometry and commutative algebra, in preparation.

[Fu] W. Fulton, Algebraic curves, Benjamin, New York, 1969.

[Ha] R. Hartshorne, Algebraic geometry, Springer-Verlag, New York, 1977.

[FAC] J. P. Serre, Faisceaux algebriques coherents, Ann. of Math. 61 (1955), 197-278.

[HSV] J. Herzog, A. Simis, and W. V. Vasconcelos, Koszul homology and blowing-up rings, Commutative Algebra, Proc. Trento Conference, 1981, pp. 79-169. ITALIA

Department of Mathematics, Cornell University, Ithaca, New York 14853 